\title{
Movement behavior of a spider on a horizontal surface
}

\author{
WANG Zhou Yi ${ }^{1,2}$, WANG JinTong $^{1,2}$, JI AiHong $^{1}$, LI HongKai $^{1,2}$ \& DAI ZhenDong ${ }^{1 *}$ \\ ${ }^{1}$ Institute of Bio-inspired Structure and Surface Engineering, Nanjing University of Aeronautics and Astronautics, Nanjing 210016, China; \\ ${ }^{2}$ College of Mechanical and Electrical Engineering, Nanjing University of Aeronautics and Astronautics, Nanjing 210016, China
}

Received October 14, 2010; accepted May 27, 2011

\begin{abstract}
Studying the locomotive behavior of animals has the potential to inspire the design of the mechanism and gait patterns of robots ("bio-inspired robots"). The kinematics characteristics of a spider (Ornithoctonus huwena), including movement of the legs, movement of the center of mass (COM) and joint-rotation angle, were obtained from the observation of locomotion behaviors recorded by a three-dimensional locomotion observation system. Our results showed that one set of the stance phase consists of four legs, which were leg- 1 and leg-3 on one side and leg- 2 and leg-4 on the other side. Additionally, two sets of the stance phase comprised eight legs alternately supporting and driving the motion of the spider's body. The spider primarily increased its movement velocity by increasing stride frequency. In comparison to other insects, the spider, O. huwena, has superior movement stability. The velocity and height of COM periodically fluctuated during movement, reaching a maximum during alternation of leg phase, and falling to a minimum in the steady stance phase. The small change in deflection angle of the hind-leg was effective in driving locomotion, whereas each joint-rotation angle of the fore-leg changed irregularly during locomotion. This research will help in the design of bio-inspired robots, including the selection of gait planning and its control.
\end{abstract}

locomotive behavior, Ornithoctonus huwena, bio-inspired robot, joint-rotation angle, three-dimensiond locomotion observation system

Citation: Wang Z Y, Wang J T, Ji A H, et al. Movement behavior of a spider on a horizontal surface. Chinese Sci Bull, 2011, 56: 2748-2757, doi: $10.1007 / \mathrm{s} 11434-011-4584-\mathrm{y}$

Locomotive behavior and physiological characteristics of animals depend heavily on their environmental circumstances, which create morphological and physiological evolutionary pressures on animals for efficiency, flexibility and adaptability [1]. Animals have evolved different locomotive behaviors to adapt to their specific living environment. Thus, animals are far superior, in terms of stability, agility, robustness, environmental adaptability and efficiency, to robots [2]. Research on the locomotive behaviors of animals can reveal characteristics of animal motion that could inspire engineers to develop biomimetic robots with advanced performance [3-7].

The locomotive behavior of two-legged, four-legged and six-legged animals has been studied widely [8-15]. The application of spider poison and the mechanical properties

*Corresponding author (email: zddai@ nuaa.edu.cn) of spider webs had been studied widely [16-19]. In research on spider kinematics, Land found that stepping movements during turns made by a tarantula were mediated by nerves of the lateral eyes [20]. Bromhall uncovered the close relationship between spider heart-rates and locomotion [21]. Shultz compared the kinematics of terrestrial and semiaquatic spiders [22]. Wang measured the reaction force of each spider leg on a level surface, and discussed the function of different legs compared with those of four-legged and six-legged animals [23]. The movement control system of a spider-like robot was studied by Ohnish et al. [24]. Gasparetto et al. established a motion model of a spider and performed a simulation analysis [25].

The spider $O$. huwena is a burrowing eight-legged animal that generally inhabits a complex environment (such as grass or a burrow) [26], and has a superb ability to climb over obstacles and to move even if it has damaged legs. 
This spider provides a useful biological template to use in the design of a reliable robot for space exploration. However, many issues, such as the relationship between redundant stance phase and stability, function of each leg, locus of center of mass (COM), change in joint-rotation angle of each leg, among others, remain unclear. In this paper, we used a three-dimensional locomotion observation system to reveal the characteristics of the spider's locomotion.

\section{Materials and methods}

\subsection{Animals and facilities}

The spider $O$. huwena is composed of a cephalothorax, abdomen and limbs. Limbs include a pair of chelicerae and four pairs of walking legs. Each leg can be further separated into the coxa, trochanter, femur, patella, tibia, metatarsus and tarsus [22]. Three spiders (Figure 1(a)) were obtained from Guangxi, China and were maintained separately in cages under a natural light cycle and a constant temperature of $25 \pm 2^{\circ} \mathrm{C}$ (mean \pm s.d.). Spiders were fed with fresh pig's liver and water. The physical parameters of the spiders are listed in Table 1.

A three-dimensional locomotion observation system, consisting of a test tunnel with two mirrors on opposite sides, a high speed camera (Mikrotron, MC1311, Germany) vertically-fixed to the aisle for recording the images from a birds-eye view and two side-views, as well as a computer, were used to record the images. The test tunnel was composed of a substrate covered with graph paper $(10 \mathrm{~mm} \times 10 \mathrm{~mm})$, a transparent cover board and two mirrors positioned at $45^{\circ}$ to the test tunnel. The test tunnel was wide enough to not affect the free swing of the spider's legs. The high speed camera was supported with a tripod and connected to a computer to set the frame frequency, pixels, start and stop times (frame frequency: $100 \mathrm{fps}$, pixel: $1024 \times 1024$; Figure $1(\mathrm{~b}))$.

\subsection{Methods}

Four pairs of walking legs were defined as L1, L2, L3, L4 (left side) and R1, R2, R3, R4 (right side; Figure 2(a)). To simplify the description, we assumed that the underside of the tunnel in the three-dimensional locomotion observation system represented the horizontal plane (body plane), meanwhile the symmetrical plane of the body represented the sagittal plane. Finally, the leg-section plane represented the plane through which contact is made between a leg and the substrate as well as the joint of the coxa and the body (perpendicular to the body plane). During movement, there was no change in the contact between the tarsus and the substrate, or the angle between the tarsus and metatarsus. Thus, the influence of the tarsus on joint-rotation angle was ignored, and the trochanter and patella were simplified to represent a single joint. The joints between the coxa and femur, the femur and tibia and the tibia and metatarsus were defined as joint-a, joint-b and joint-c, respectively. The deflection angle $(\theta)$ is the angle between the leg-section plane

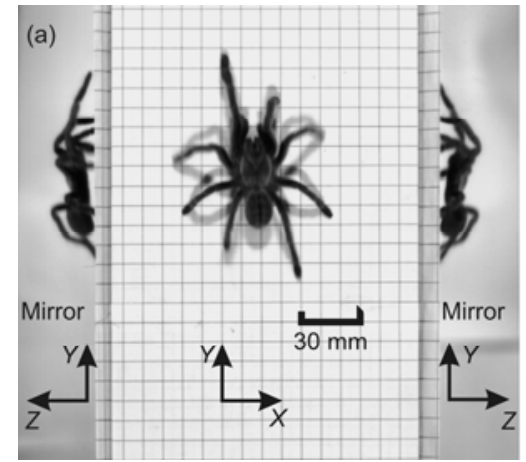

(b)

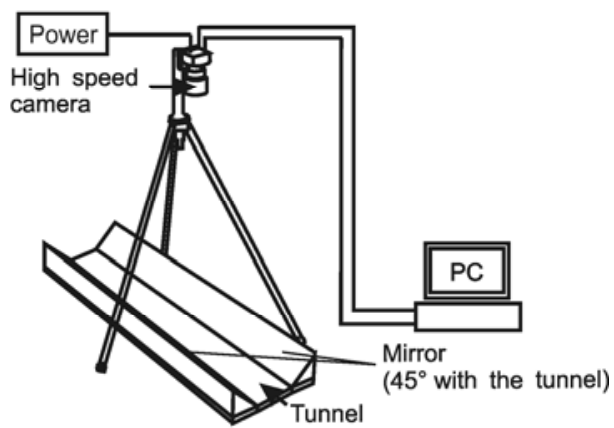

Figure 1 (a) Image record; (b) three-dimensional locomotion observation system.

Table 1 Body characteristics for $O$. huwena

\begin{tabular}{|c|c|c|c|c|c|c|c|c|}
\hline & \multirow{2}{*}{$\begin{array}{l}\text { Weight } \\
(\mathrm{g})\end{array}$} & \multirow{2}{*}{$\begin{array}{l}\text { Body length } \\
(\mathrm{mm})\end{array}$} & \multirow{2}{*}{$\begin{array}{l}\text { Cephalothorax } \\
\text { length (mm) }\end{array}$} & \multirow{2}{*}{$\begin{array}{l}\text { Cephalothorax width } \\
(\mathrm{mm})\end{array}$} & \multicolumn{4}{|c|}{ Leg length (mm) } \\
\hline & & & & & Leg-1 & Leg-2 & Leg-3 & Leg-4 \\
\hline Sample 1 & 5.41 & 44.4 & 24.1 & 15.9 & 57.1 & 43.8 & 42.0 & 48.8 \\
\hline Sample 2 & 6.35 & 53.4 & 30.6 & 17.6 & 62.8 & 46.7 & 44.2 & 56.5 \\
\hline Sample 3 & 5.02 & 40.3 & 21.4 & 15.7 & 51.1 & 43.3 & 36.5 & 45.4 \\
\hline Mean \pm s.d. & $5.59 \pm 0.56$ & $46.0 \pm 5.5$ & $25.4 \pm 3.9$ & $16.4 \pm 0.9$ & $57.0 \pm 4.8$ & $44.6 \pm 1.5$ & $40.9 \pm 3.2$ & $50.23 \pm 4.6$ \\
\hline
\end{tabular}



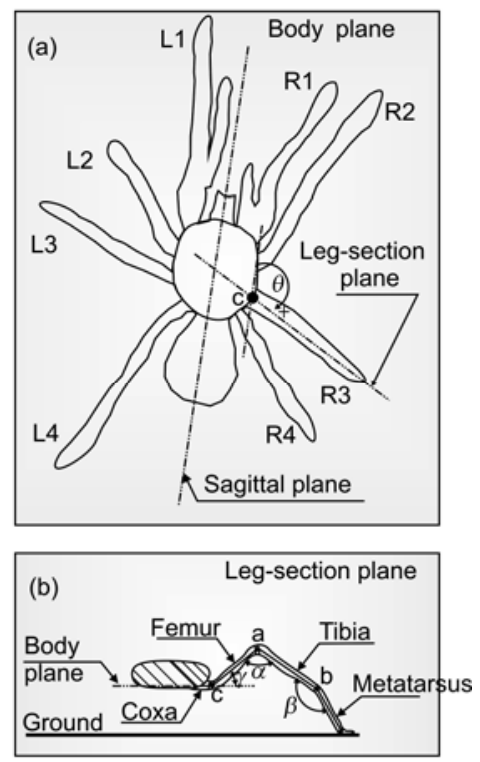

Figure 2 Rotation angles defined each joint in a different plane. (a) The rotation angle of joint-c was the deflection angle in the body plane; (b) the rotation angles of joint-a, joint-b and joint-c were the femur-tibia angle $(\alpha)$, tibia-metatarsus angle $(\beta)$ and the lifting angle $(\gamma)$, respectively, in the leg-section plane.

and the sagittal plane, which reflects the angle change of joints-c in the body plane (Figure 2(a)). The angle between the femur and tibia, the tibia and metatarsus and the tibia and metatarsus was defined as the femur-tibia angle $(\alpha)$, the tibia-metatarsus angle $(\beta)$, and the lifting angle $(\gamma)$, respectively. These reflect change in angle of joint-a, joint-b and joint-c in the leg-section plane (Figure 2(b)).

(i) Data processing. Coordinates (in the form of pixels) of each point on the images were extracted by Sigma Scan Pro (Systat Software Inc., CA). From these coordinates, information on locomotion parameters, such as stride length, stride frequency, duty factor, locus of COM, and continuous angle of joint, could be derived. The first pair of compound eyes was taken as the reference point to calculate the velocity of motion. Errors were accrued in the process of calculating the coordinates from images as evidenced by the fluctuation of the locomotion parameters. Thus, data obtained from images were filtered using digital tools (Biotest, Germany), which remove jitter while retaining the original curve of the peak. The filtered data were compared with the original data using analysis of variance.

(ii) Statistics. During movement, locomotive behaviors of the experimental animal $O$. huwena might be influenced by many factors, such as body weight, stress and environmental conditions. Thus, statistical analyses must consider these other factors to reveal the role of locomotion mechanics. We compared the differences between groups using $t$-tests, setting a statistical significance threshold of $P=0.05$, and investigated the relationship between locomotion parameters and velocity with regression analysis. All of the tested data are presented as means \pm standard deviations (mean \pm s.d.). Statistical analyses were performed using SPSS software (SPSS Inc., Chicago, IL).

\section{Results}

The legs of $O$. huwena were symmetrical. The various locomotive parameters (stride frequency, stride length, jointrotation angle, etc.) of corresponding legs of the left and right sides were not significantly different. Therefore, the parameters of corresponding legs on both sides were combined for analysis.

\subsection{Gait characteristic parameters}

The velocity of animals is the product of stride frequency and stride length. The results showed that both stride frequency and stride length was significantly related with velocity of $O$. huwena. The stride length decreased while the stride frequency increased with increasing velocity. As the influence of stride length on velocity was small, spiders apparently primarily control their velocity by changing their stride frequency (Figure 3(a), Table 2). The velocity was related to step length of each leg. The amplitude of step length of leg-3 was the largest with a change in velocity, while the amplitude of step length of leg-1 was smallest. The duty factors of leg-2, leg-3 and leg-4 were significantly affected by velocity and decreased with increasing velocity. When $O$. huwena moved faster, the swing phase time was increased and the stance phase time was decreased (Figure 3(b)-(e), Table 2).

\subsection{Overview of legs}

The eight legs of $O$. huwena were divided into two sets of stance legs (one set: L1, R2, L3, R4; another set: R1, L2, R3, L4), which support the body moving forward in an alternating fashion. The results showed that the gait of $O$. huwena did not change with a change in velocity. To follow the location of $O$. huwena in a whole period, we evaluated a discrete periodic sample (a gait cycle starts from R4 falling to the ground and ends the next time R4 falls to the ground) from the images. One whole period includes two stable moving states (states 1 and 5) and six alternate states of legs (states 2, 3, 4, 6, 7 and 8). The mobile order of legs was the same during alternate states of the stance phase. Form state 1 to state 2, the COM moved from the latter supporting triangular area formed by lines joining three contacted points between the substrate and legs (R2, L3, R4) into the former supporting triangular area formed by three contacted points between the substrate and legs (L1, R2, L3). Leg-L2 and leg-R3 fell into the supporting triangular area. Thus the body was supported simultaneously by leg-L1, -L2, -R2, $-\mathrm{L} 3,-\mathrm{R} 3$ and $-\mathrm{R} 4$ at state 2 . Subsequently, leg-R4 left the 

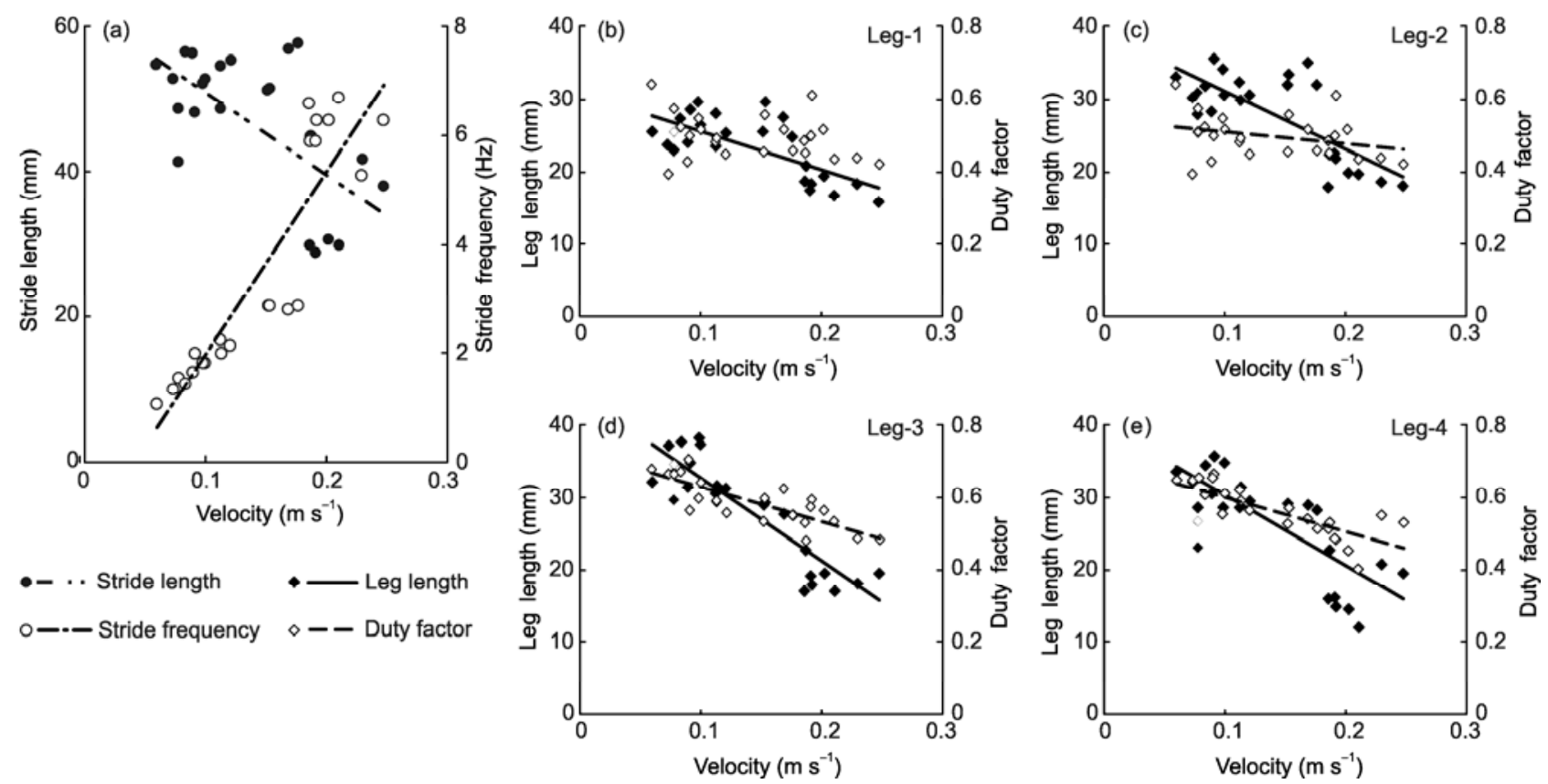

Figure 3 Stride length (a), stride frequency (a), duty factors (b, c, d, e) and leg lengths (b, c, d, e) $v s$ velocity for a spider, $O$. huwena moving on a horizontal surface. (a) Stride length and stride frequency $v s$ velocity for $O$. huwena moving on a horizontal surface; (b)-(e) duty factor and leg length of each leg $v s$ velocity for $O$. huwena moving on a horizontal surface.

Table 2 Parameters of gait and their relationship with velocity

\begin{tabular}{|c|c|c|c|c|c|c|c|c|}
\hline & \multirow{2}{*}{ Leg } & \multirow{2}{*}{ Mean \pm s.d. } & \multirow{2}{*}{$N$} & \multicolumn{5}{|c|}{ Regression analysis with velocity } \\
\hline & & & & $a$ & $b$ & $R_{2}$ & $F$ & $P$ \\
\hline Stride length & & $48.24 \pm 10.24$ & 27 & -115.42 & 64.561 & 0.4 & 14.667 & $<0.001$ \\
\hline Stride frequency & & $3.43 \pm 2.06$ & 27 & 33.4 & -1.3539 & 0.669 & 50.5 & $<0.001$ \\
\hline \multirow{4}{*}{ Duty factor } & 1 & $0.49 \pm 0.06$ & 25 & - & - & 0.083 & 2.34 & 0.163 \\
\hline & 2 & $0.59 \pm 0.07$ & 25 & -0.9147 & 0.7187 & 0.5236 & 25.2788 & $<0.001$ \\
\hline & 3 & $0.59 \pm 0.07$ & 25 & -0.9647 & 0.7263 & 0.6634 & 45.3304 & $<0.001$ \\
\hline & 4 & $0.52 \pm 0.07$ & 25 & -0.9528 & 0.6951 & 0.6051 & 35.2426 & $<0.001$ \\
\hline \multirow{4}{*}{ Leg length } & 1 & $23.33 \pm 4.25$ & 25 & -52.19 & 30.72 & 0.4743 & 19.849 & $<0.001$ \\
\hline & 2 & $27.80 \pm 5.97$ & 25 & -79.585 & 39.061 & 0.5602 & 28.0227 & $<0.001$ \\
\hline & 3 & $27.88 \pm 7.24$ & 25 & -114.82 & 44.113 & 0.792 & 83.7692 & $<0.001$ \\
\hline & 4 & $26.33 \pm 7.67$ & 25 & -98.424 & 40.249 & 0.5196 & 23.7952 & $<0.001$ \\
\hline
\end{tabular}

ground as leg-L4 fell into the former supporting triangular area, and then the supporting area of the convex quadrilateral became a supporting triangular area in which six legs were located (state 3). From state 3 to state 4, leg-L1, -R2 and -L3 left the ground continually. Thereby the body was supported simultaneously by leg-L2, -R3 and -L4 at state 4, and the COM was located in the latter supporting triangle. Finally, the spider moved into the stable moving state by the leg-R1 falling to the ground (state 5), completing one alteration of the spider's leg. The next leg alteration began (states 6, 7 and 8) and eventually returned to state 1 (Figure 4(a) and (b)).

\subsection{Fluctuation of the COM}

At the beginning of a cycle, the body was firmly supported by four legs, the COM was lower and velocity was relatively low (Figure 4(c)). During forward motion, the phase of each leg began to alternate (states 2, 3 and 4). During the initial contact period, the legs changed from the swing phase to the stance phase, thereby gradually slowing the velocity and lowering the height of the COM. The height and velocity of the $\mathrm{COM}$ reduced to a minimum at the same time and the COM crossed the frontier of the latter and former supporting triangular areas. Subsequently, the spider 
(a)
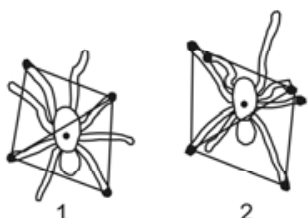

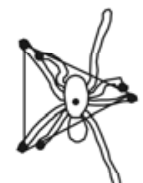

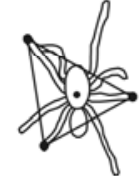

4

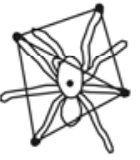

5

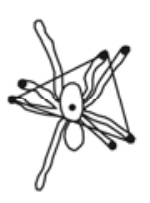

6

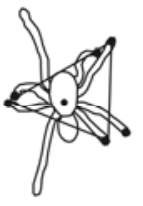

7

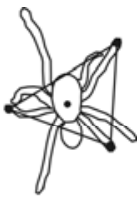

8

(b)
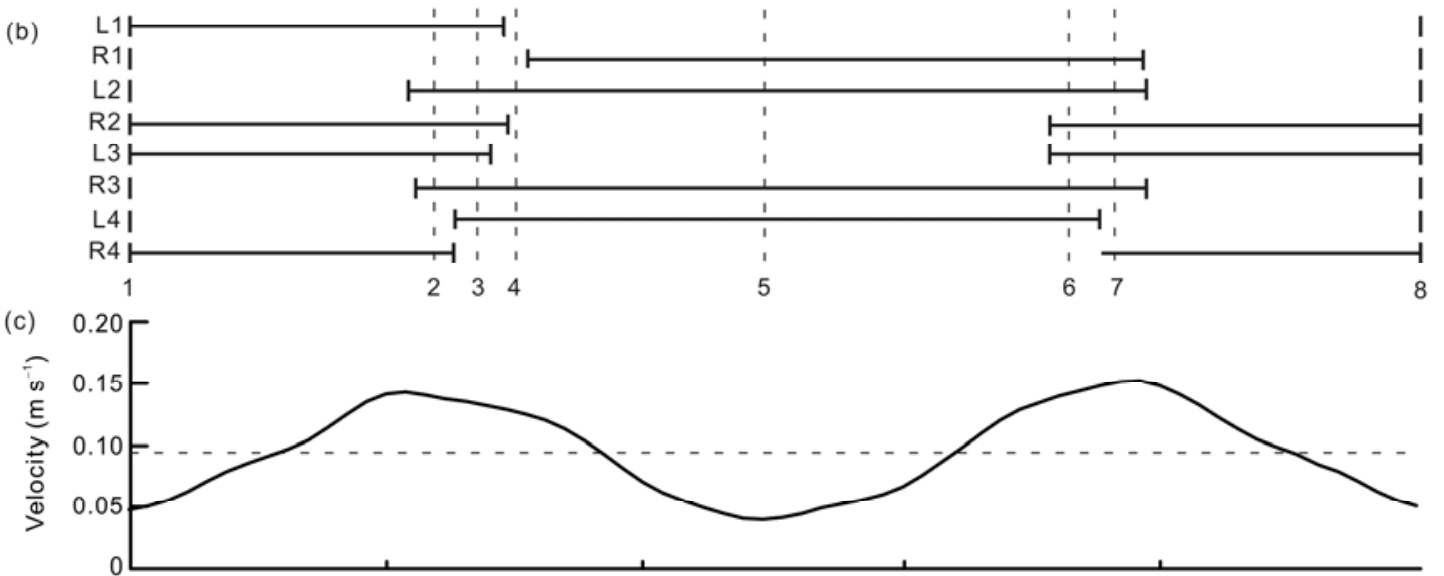

(d)

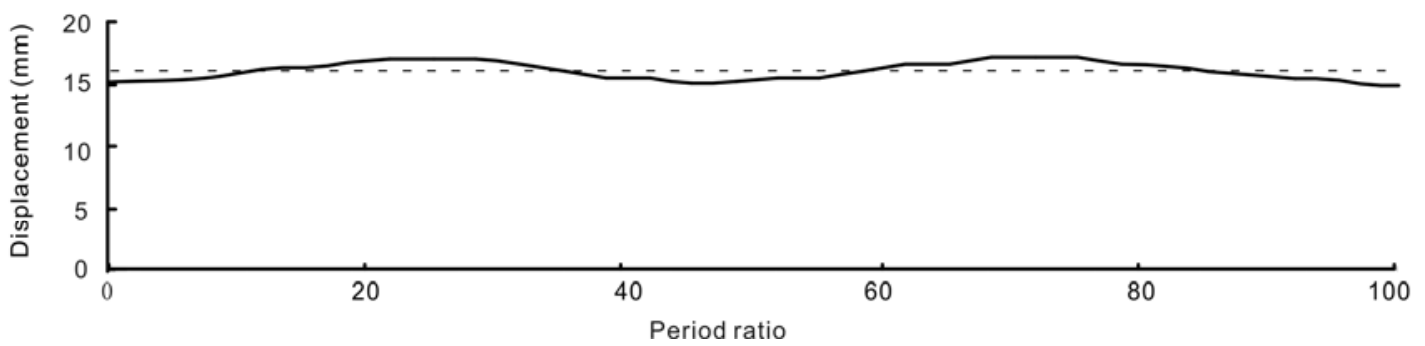

Figure 4 (a) Tracing of a spider $O$. huwena moving on a horizontal surface; (b) gait pattern and timing of stance and swing for each leg; (c) velocity curve of center of mass in body plane; (d) displacement curve of center of mass in normal direction.

began to accelerate and rapidly finished alteration of phase. The cyclical swing frequencies of the COM velocity and height were twice as high in comparison to those of the stride frequency (Figure 4(d)).

\subsection{Joint-rotation angle}

The temporal joint angular curves are intuitive ways to depict the movement of the leg during animal locomotion, and can be used to improve the design of bio-robots, especially in terms of the planning of gait and coordinating locomotion. Data of variations of joint-rotation angle movement on a horizontal surface $\left(0.095 \mathrm{~m} \mathrm{~s}^{-1}\right)$ were selected for analysis. Extremes and ranges of joint-rotation angles are listed in Table 3. The period of locomotion was normalized to allow for comparison with different experiments. Joint angular curves of each leg during a gait cycle are shown in Figure 5. One stance phase and one swing phase are included in one gait cycle. The femur-tibia angle ( $\alpha$, dash-dot line), the tibia-metatarsus angle ( $\beta$, dashed line), the lifting angle $(\gamma$, two-dot chain line) and the deflection angle ( $\theta$, solid line) curves all achieved extremes during alternating phases.

(i) Femur-tibia angle $\alpha$. Variations of the femur-tibia angle showed obvious differences between each leg. The femur-tibia angle of leg-1 had no obvious change during the stance phase. However, during the swing phase, the femur-tibia angle of leg-1 passed through three periods: decreasing, maintaining, and increasing. Simply put, joint-a of leg-1 changed frequently during the swing phase. The femur-tibia angle of leg-2 increased and then decreased during the swing phase, with little fluctuation. Joint-a of leg-2 had one extension and one flexion during the swing phase. The femur-tibia angle of leg-3 slightly decreased during a later period of the swing phase. Joint-a of leg-3 extended and flexed one time during an earlier period of the swing phase, and it always remained unfolded until leg-3 touched the ground. Joint-a of leg-4 extended during the stance phase and flexed during the swing phase. Thus, the femurtibia angle of leg-4 increased during the stance phase and decreased during the swing phase (Figure 5). 

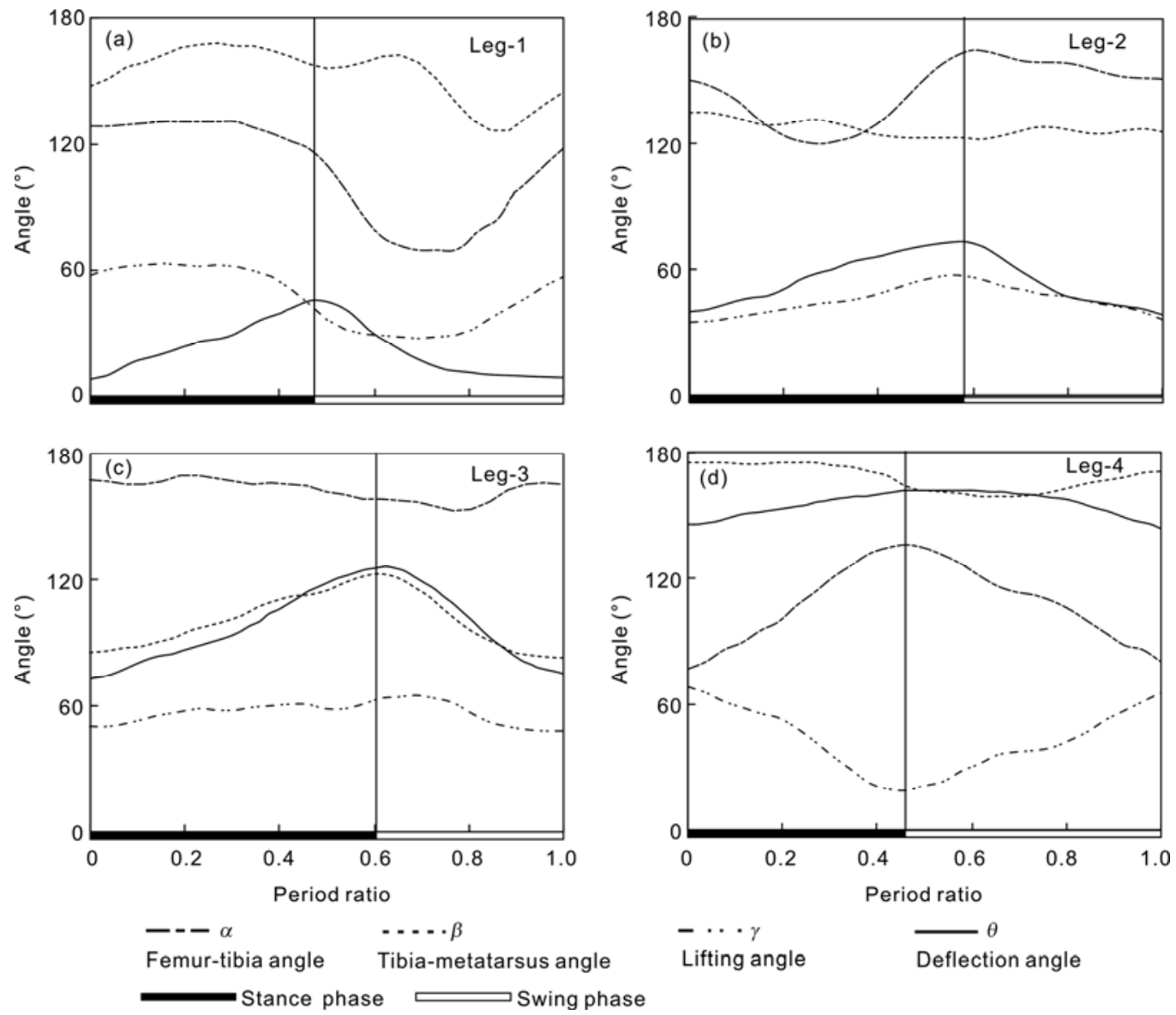

Figure 5 Change curve of each joint-rotation angle in one cycle for a spider $O$. huwena moving on a horizontal surface. (a)-(d) change curves of each joint-rotation angle of leg-1, leg-2, leg-3 and leg-4 in one cycle, respectively.

(ii) Tibia-metatarsus angle $\beta$. The tibia-metatarsus angle of leg-1 constantly increased during the stance phase. During the swing phase, the tibia-metatarsus angle of leg-1 underwent three periods: increasing, decreasing and increasing, thus resulting in one peak and one wave trough in the curves. Joint-b of leg- 2 rotated unobtrusively, and thus the tibia-metatarsus angle of leg- 2 only slightly changed. Joint-b of leg-3 extended and flexed only one time during a whole gait cycle. The tibia-metatarsus angle of leg-3 increased during the stance phase and reduced to the initial angle during the swing phase. The tibia-metatarsus angle curve of leg-4 had one wave trough during the swing phase (Figure 5).

(iii) Lifting angle $\gamma$. Change in the lifting angle and femur-tibia angle of leg-1 had a similar trend. Indeed, joint-c of leg-1 had similar rotation as that of joint-a in the leg-section plane. The lifting angle of leg- 2 and leg- 3 increased during the stance phase and achieved a peak at the phase switch, after which it consistently decreased. Thus, joint-c had one extension and one flexion during a whole gait cycle. The lifting angle of leg-4 increased during the stance phase and decreased during the swing phase. Thus, joint-c of leg-4 flexed during the stance phase and extended during the swing phase (Figure 5).

(iv) Deflection angle $\theta$. Change in the deflection angle curves of each leg showed a similar trend in the body plane, which increased during the stance phase and decreased during the swing phase. Thus, joint-c of each leg extended during the stance phase and flexed during the swing phase. The amplitude change of the deflection angle of leg- 3 was largest, and that of leg-4 was the smallest. Leg-1 and leg-2 had similar amplitudes of change in the deflection angle (Figure 5).

\section{Discussion}

Research focusing on the function and locomotion characteristics of legs of the large spider $O$. huwena can be applied to the development of a spider-robot, especially in the design of the structure of legs and planning of gait pattern.

\subsection{Locomotion of the COM}

One set of the stance phase consists of leg- 1 and leg- 3 on one side and leg- 2 and leg-4 on the other side of the body. 
Two sets of the stance phases comprise eight legs alternately supporting and driving the motion of the spider's body. A gait cycle starts from leg-4 flexing and falling to the ground, and ends with leg-4 extending and lifting off the ground. Each joint of leg-4 under the stance phase extends constantly to release energy and move the body along the direction of leg-4 (Figure 6(a)). However, the body moves forward along the direction of locomotion as an independent rigid body, thus the driving efficiency of leg- 4 is reduced by the angle between the directions of driving and the locomotion. The observations showed that the spider adjusted the defection angles of each leg in an effort to reduce hindrance of function (Figures 4(a), 6(a)). Moreover, the change in the defection angle of leg-4 was obviously smaller than that of leg-2 and leg-3 during a whole cycle. This locomotive method can make the main driving force provided by leg-4 more efficient to drive the body and improve the driving efficiency of movement (Figure 5, Table 3).

The contact point between the leg and substrate can be described as the immobile point during the stance phase, such as $A_{L 1}, A_{L 3}, A_{R 2}$ and $A_{R 4}$. A supporting quadrilateral area was made up of contact points $\left(\mathrm{A}_{\mathrm{L} 1}, \mathrm{~A}_{\mathrm{L} 3}, \mathrm{~A}_{\mathrm{R} 2}\right.$ and $\left.\mathrm{A}_{\mathrm{R} 4}\right)$ during one set of the stance phase. At the beginning, the $\mathrm{COM}$ was located in the latter supporting triangular area $\left(A_{L 3} A_{R 2} A_{R 4}\right)$. As the leg-R4 moved forward, the COM moved across the line formed by $A_{L 3}$ and $A_{R 2}$ into the former supporting triangular area (Figure 6(a)). The spider's continuing supporting quadrilateral areas, as shown in Figure 3(a), were arranged in order of gait and time. Thus, the movement locus of the COM during locomotion was Zshaped (Figure 6(b)). The displacement of the body caused by rotation of joint-c was minor in the body plane. Thus, the body only slightly rotated and its main locomotion was a parallel shift along the direction of general movement. To accomplish this parallel shift, legs on both sides of the body must undergo joint rotation, which must be different in the leg-section plane (Table 3, Figure 5) to generate differences in displacement. Meanwhile, the height and velocity of the COM continually fluctuate during joint rotation (Figure 4(c) and (d)).

At the beginning of each step (cycle ratio: $0.00 \pm 0.00$ $0.16 \pm 0.02$ ), the leg touched the substrate, and the velocity and height of the COM were reduced. During the follow-up period (cycle ratio: $0.16 \pm 0.02-0.25 \pm 0.03$ ), the COM was raised and accelerated. The locomotion of a single leg could be simplified to a spring model during the stance phase [27].

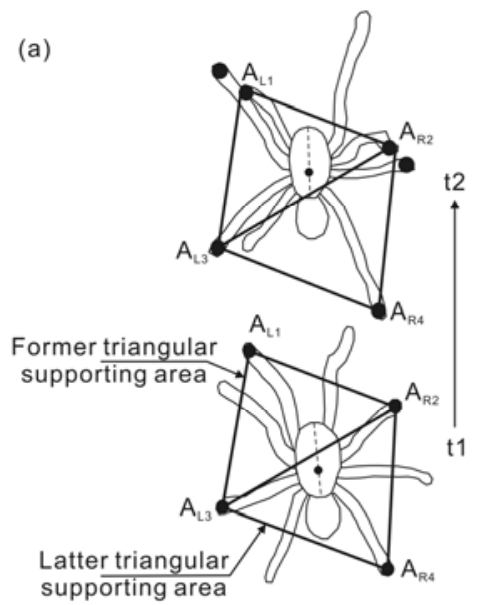

(b)

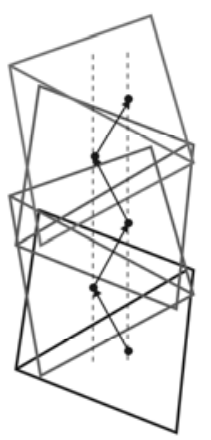

Figure 6 (a) The center of mass position of single gait cycle in the body plane of the spider. (b) Locus of center of mass in body plane for a spider $O$. huwena moving on a horizontal surface.

Table 3 Extremes and ranges of each joint-rotation angle for O. huwena $\left(^{\circ}\right)$

\begin{tabular}{|c|c|c|c|c|c|c|c|c|c|}
\hline \multirow{2}{*}{ Joint-rotation angle } & & \multicolumn{2}{|c|}{ Leg-1 } & \multicolumn{2}{|c|}{ Leg-2 } & \multicolumn{2}{|c|}{ Leg-3 } & \multicolumn{2}{|c|}{ Leg-4 } \\
\hline & & Stance phase & Swing phase & Stance phase & Swing phase & Stance phase & Swing phase & Stance phase & Swing phase \\
\hline \multirow[t]{3}{*}{ Femur-tibia angle $\alpha$} & Max. & 131.00 & 121.30 & 165.01 & 164.46 & 171.14 & 167.41 & 135.37 & 132.18 \\
\hline & Min. & 121.30 & 69.37 & 120.83 & 152.45 & 159.01 & 154.10 & 77.77 & 76.59 \\
\hline & Ra. & 9.70 & 51.92 & 44.19 & 12.01 & 12.13 & 13.31 & 57.60 & 55.59 \\
\hline \multirow{2}{*}{ Tibia-metatarsus angle $\beta$} & Min. & 147.99 & 126.26 & 123.16 & 126.07 & 86.83 & 86.83 & 168.53 & 158.58 \\
\hline & Ra. & 19.76 & 35.92 & 12.66 & 2.70 & 36.74 & 35.46 & 6.79 & 12.54 \\
\hline \multirow[t]{2}{*}{ Lifting angle $\gamma$} & Max. & 63.32 & 56.84 & 58.18 & 58.18 & 65.20 & 66.11 & 68.04 & 65.25 \\
\hline & Min. & 51.59 & 27.46 & 35.71 & 35.54 & 51.52 & 48.87 & 19.64 & 18.72 \\
\hline \multirow[t]{3}{*}{ Deflection angle $\theta$} & Max. & 44.94 & 44.67 & 74.46 & 74.30 & 125.87 & 127.01 & 162.01 & 161.58 \\
\hline & Min. & 9.18 & 9.55 & 41.13 & 39.20 & 73.94 & 76.63 & 145.85 & 143.27 \\
\hline & Ra. & 35.76 & 35.12 & 33.34 & 35.10 & 51.92 & 50.38 & 16.16 & 18.31 \\
\hline
\end{tabular}


In the initial contact period, the velocity and height of the COM was low, the energy and potential energy was stockpiled by the spring of the leg, which was compressed. After reaching the limit of compression, the energy stockpiled by the spring of the leg was released, the COM began to accelerate and its height was raised. Thus, the spider had significant energy in the later period of each step because of the release of spring potential energy to maintain stable movement and accomplish alternation of phase.

Taking into account all the factors above, the locomotive locus of the COM in the body and sagittal plane were combined for analysis. The locomotion of the COM could be simplified as a rolling along the axis formed by the tips of leg-2 and leg-3 and a parallel shift along the driving direction of leg-4, which indirectly showed locomotive wave properties and periodicity. The above description could be applied in the design of creeping-type leg structures for spider-robots to coordinate changes in each joint and reduce unnecessary vibration as far as possible, thereby improving the locomotion system stability of the bio-robot. The springmass model clearly revealed the process by which animals recycle the energy of fluctuations in the locomotive system [27]. Thus, we could use fluctuations of spider locomotion and stockpile it as energy to improve energy efficiency in subsequent locomotion.

\subsection{Comparison of gaits in locomotion}

O. huwena can move in a complex environment, such as those with a rough substrate, and in and out of holes to prey on animals and explore the environment. The results showed that the velocity of $O$. huwena was mostly affected by stride frequency and less so by stride length. The contact time between the leg and the substrate decreased because of the increasing velocity and the decreasing duty factor. Most parts of the whole cycle comprised a stable moving state. Moreover, the body of the spider was supported by four legs during the stable moving state and more than four legs during the alternate state (Figure 3, Table 2). It is apparent that the basic locomotive trait of $O$. huwena is similar to that of the terrestrial spider [22], but obviously distinct from that of the net-knitting spider [28]. In the net-knitting spider, there is neither an obvious stable moving state nor alternate state during locomotion, and there are always five legs under the body during the stance phase.

\subsection{Stability of locomotion}

Animals vary in their anatomy, and locomotive behavior. Therefore, there are a number of differences in locomotion gaits and stable methods among animals. Some insects, such as ants, cockroaches and the pentatomid bug Erthesina fullo, move on the surface using a triangular gait [29-31].

In 2002, Xu et al. analyzed the stability of the triangular gait of a mini bio-robot and produced a calculation method of stability redundancy for triangle gaits [32]. This calculation method was applied to a polygonal gait, and stability redundancy was defined as the minimum distance at which the COM was outside of the convex polygons formed by contacted points. Thus, the size of the stability redundancy is determined by the size of the polygon and the position of the COM. The stability redundancy is used to evaluate stability of locomotion. In comparison to six-legged insects moving in a triangular gait, the body of $O$. huwena was supported by four legs during stable moving states and the supporting area was convex quadrilateral area. Thus, under the same conditions, the stability of spiders was superior to that of insects during stable moving states. An additional leg plays an important role in improving the stability of locomotion. However, an additional leg will certainly influence the time needed for alternating legs. The velocity of $O$. huwena was lower than that of six-legged insects under the same experiential conditions. Meanwhile, the velocity of a crawling spider is low during normal foraging. It is well known that the evolution of physiological structure is closely related to environment. In nature, the daily activities of a spider do not require high- speed locomotion but rather depend on specializations, such as using venom or predation by spinning webs. However, in designing robots and other mechanical devices, we select velocity and stability of locomotion, based on the actual requirements, to optimize mechanical motion. For example, in a space expedition, the reliability and stability of a robot's locomotion are more important than the ability for fast movement.

In the present study, we observed $O$. huwena moving on a horizontal plane to analyze the traditional static stability of spiders and obtain some basic kinematic information. This information provided basic understanding of the movement that can be explored in further research, such as on moving over obstacles and disturbed motion of spiders. Our findings regarding the locomotion stability of $O$. huwena were identified during the early stages of exploration and comprised many aspects, such as the influence of high speed on locomotion stability. Thus, to further investigate these findings, we will measure the reaction force of a leg and simultaneously record kinematic information of the spider using a dynamic measuring system that we have developed. This information will be combined to analyze the dynamic stability of a spider in locomotion and to reveal the coordinates of each leg and influences on velocity.

\subsection{Function of the leg}

The particular locomotion of $O$. huwena is ideal for living on a rough surface because of the constructed diversity of the four pairs of legs [19]. Meanwhile, this diversity would suggest that different legs have different functions during the locomotion.

Leg-1 was the longest among all the legs and its duty factor was the smallest. Before leg-1 of one side fell to the 
ground, leg-1 of the opposite side rose up. The duty factor of leg-1 was not affected by velocity (Table 2). During the stance phase, there were small changes in the femur-tibia angle, the tibia-metatarsus angle and the lifting angle of leg-1. Specifically, each joint of leg-1 slightly rotated in the leg-section plane. However, the changes in the rotational angle of each joint were larger, and thus leg-1 showed obvious extension and flexion for exploring behavior during the swing phase (Figure 4(a), Table 3).

The direction of the reaction force was opposite to the direction of locomotion [20]. Indeed, leg-1 was mainly used for leading, exploring and absorbing shocks, and it was also used for catching food in the field [33]. Leg-4 was similar to leg-1 in structure and was slightly shorter than leg 1, but showed great differences in behavior characteristics and gait parameters (step length $t$-tests, $P=0.003$, duty factor $t$-tests, $P=0.001$ ). Leg-4 finished phase switching in the middle of the whole cycle and its duty factor was about 0.5 (Table 2). During the stance phase, leg-4 continuously extended to release energy and drive the body movement forward. Leg-4 persistently flexed to store energy during the swing phase. This wide angular extension and flexion provided enough driving force for locomotion (Table 3), and the direction of this driving force was also consistent with the direction of movement [20]. In addition, the deflection angle of leg-4 showed little variation, which preserved the angle between the leg- 4 section plane and the sagittal plane in stance phase. This kinetic characteristic described above improved driving efficiency of leg-4 (Figure 5(d), Table 3). The structures of leg- 2 and leg-3 were similar, as were their gait characteristics (step length $t$-tests, $P=0.282$, duty factor $t$-tests, $P=0.753)$. Leg- 2 and leg-3 were under the supporting phase for more than half of the gait cycle and their duty factors were larger (Table 2). The structures of leg-2 and leg-3 in the middle of the body determined that the directions of the leg's extension and flexion were nearly perpendicular with the direction of locomotion and accordant with the lateral direction (Table 3). Thus, the large loading in the lateral direction could be negatively affected by leg- 2 and leg-3, which generated large lateral force. Consequently, leg-2 and leg-3 mainly supported the body away from the ground, and suffered the lateral perturbation to maintain lateral stability. Moreover, these two legs could also be helpful for locomotion (Figure 5(b) and (c)).

The functions of healthy spider legs are discussed above, but in nature, a spider can move even if it has lost one or more of its legs. It is unclear how such coordination and compensation for the functions of damaged legs is accomplished. Thus, another study should assess this problem, which would will help in the design of robots and gait planning. For example, if one or more legs are destroyed, an eight-legged robot could redesign its gait and thus continue its assignment, without becoming immobilized. Such an invention would dramatically help improve a robot's working ability, reliability and cost.
This work was supported by Funding for Outstanding Doctoral Dissertation in Nanjing University of Aeronautics and Astronautics (BCXJ10-10), the National Hi-Tech Research and Development Program of China (2007AA04Z201), and the National Natural Science Foundation of China (60910007, 31070344 and 50975140).

1 Zaaf A, Damme R V, Herrel A, et al. Spatio-temporal gait characteristics of level and vertical locomotion in a ground-dwelling and a climbing gecko. J Exp Biol, 2001, 204: 1233-1246

2 Dickinson M H, Farley C T, Full R J, et al. How animals move: An integrative view. Science, 2000, 288: 100-106

3 Sirirak T N P, Tai Y C, Ho C M, et al. Microbat: A palm-sized electrically powered ornithopter. In: Workshop on Biomorphic Robotics, 2000, 8: 14-16

4 Wang M, Zang X Z, Fan J Z, et al. Biological jumping mechanism analysis and modeling for frog robot. J Bio Eng, 2008, 5: 181-188

5 Crespi A, Badertscher A, Guignard A, et al. Amphibot I: An amphibious snake-like robot. Rob Auto Sys, 2005, 50: 163-175

6 Kato N. Control performance in the horizontal plane of a fish robot with mechanical pectoral fins. IEEE J Oceanic Eng, 2000, 25: 121-129

7 Collins S, Ruina A, Tedrake R, et al. Efficient bipedal robots based on passive-dynamic walkers. Science, 2005, 307: 1082-1085

8 Manter J T. The dynamics of quadrupedal walking. J Exp Biol, 1938, 15: 522-540

9 Pridmore P A. Trunk movements during locomotion in the marsupial monodelphis domestica (Didelphidae). J Morphol, 1992, 211: 137-146

10 Coppenolle V I, Aerts P. Terrestrial locomotion in the white stork (Ciconia ciconia): spatio-temporal gait characteristics. Animal Bio, 2004, 54: 281-292

11 Wittlinger M, Wenner R, Wolf $\mathrm{H}$. The desert ant odometer: A stride integrator that accounts for stride length and walking speed. J Exp Biol, 2006, 210: 198-207

12 Anderson F C, Pandy M G. Dynamic optimization of human walking. J Biomech Eng, 2001, 123: 381-390

13 Full R J, Tu M S. Mechanics of a rapid running insect: Two-, four-, and six-legged locomotion. J Exp Biol, 1991, 156: 215-231

14 Chen J J, Peattie A M, Autumn K, et al. Differential leg function in a sprawled-posture quadrupedal trotter. J Exp Biol, 2006, 209: 249-259

15 Li H K, Dai Z D, Shi A J, et al. Angular observation of joints of geckos moving on horizontal and vertical surfaces. Chinese Sci Bull, 2009, 54: 592-598

16 Liang S P, Qin Y B, Zhang D Y, et al. Biological characterization of spider (Ornithoctonus huwena) crudevenom (in Chinese). Zoolog Res, 1993, 14: 60-65

17 Fei R, Yang Y, Zhang L J, et al. Advance research and application of spider venom (in Chinese). J Jilin Univer (Med Ed), 2004, 30: 994-996

18 Madsen B, Shao Z Z, Vollrath F. Variability in the mechanical properties of spider silks on three levels: Interspecific, intraspecific and intraindividual. Int J Biol Macromol, 1999, 24: 301-306

19 Oroudjev E, Soares J, Arcidiacono S, et al. Segmented nanofibers of spider dragline silk: Atomic force microscopy and single-molecule force spectroscopy. Proc Natl Acad Sci USA, 2002, 99: 6460-6465

20 Land M F. Stepping movements made by jumping spiders during turns mediated by the lateral eyes. J Exp Biol, 1971, 57: 15-40

21 Bromhall C. Spider heart-rates and locomotion. J Comp Physiol B, 1987, 157: 451-460

22 Shultz J W. Walking and surface film locomotion in terrestrial and semi-aquatic spiders. J Exp Biol, 1987, 128: 427-444

23 Wang Z Y, Wang J T, Ji A H, et al. The measurement of spider's locomotion reaction force (in Chinese). Prog Nat Sci, 2009, 19: 883-888

24 Ohnishi T, Asakura T. Walking behavior of spider-robot with adaptation of environment information. In: SICE 2004 Annual Conference, Sapporo: Society of instrument and control engineers, 2005, 3: 
1999-2003

25 Gasparetto A, Vidoni R, Seidl T. Passive control of attachment in legged space robots. Appl Bionics Biomech, 2010, 7: 69-81

26 Zhu M S, Song D X. Taxonomic study on Selenocosmia huwena (Araneae: Theraphosidae: Ornithoctoninae) (in Chinese). J Hebei Univ (Nat Ed), 2000, 20: 53-56

27 Cavagna G A, Heglund N C, Taylor C R. Mechanical work in terrestrial locomotion: Two basic mechanisms for minimizing energy expenditure. Am J Physiol Regul Integr Comp Physiol, 1977, 233: R243-261

28 Takanobu H, Motegi S, Sanada K, et al. Spider based octopod. Nippon Robotto Gakkai Gakujutsu Koenkai Yokoshu, 2004, 22: 1F21

29 Reinhardt L, Weihmann T, Blickhan R. Dynamics and kinematics of ant locomotion: Do wood ants climb on level surfaces? J Exp Biol, 2009, 212: 2426-2435

30 Ting L H, Blickhan R, Full R J. Dynamic and static stability in hexapedal runners. J Exp Biol, 1994, 197: 251-269

31 Ji A H, Yan H B , Shen H, et al. Three-dimensional contact force measuring during erthesina fullo locomoting on positive/zero surface (in Chinese). J Nanjing Univer Aeron Astron, 2007, 39: 80-83

32 Xu X Y, Yang G Z, Ding G Q. Research on miniature hexapod bio-robot and its tripod gait (in Chinese). Opt Precision Engineer, 2002, 10: 392-396

33 Wang Z, Zeng B P, Wang W B, et al. Study on the biological characteristics of Ornithoctonus Huwena (in Chinese). J Beihua Univer (Nat Sci), 2002, 3: 298-299

Open Access This article is distributed under the terms of the Creative Commons Attribution License which permits any use, distribution, and reproduction in any medium, provided the original author(s) and source are credited. 